\title{
Modelo Dinámico de la Zona de Contacto en Prensas de Máquinas Papeleras
}

\author{
Francisco A. Saavedra y Pedro N. Saavedra \\ Departamento de Ingeniería Mecánica, Universidad de Concepción, Casilla 53-C, Concepción-Chile \\ (e-mail: psaavedr@udec.cl).
}

Recibido Dic. 13, 2012; Aceptado Feb. 05, 2013; Versión final recibida Mar. 22, 2013

\begin{abstract}
Resumen
El objetivo del presente trabajo es proporcionar los fundamentos físicos que permitan diagnosticar con mayor confiabilidad la condición de máquinas papeleras para su buen mantenimiento. El diagnóstico de la condición mecánica del paño y los rodillos de la sección de prensas en estas máquinas mediante el clásico análisis de vibraciones es complejo, y por ello el mantenimiento se realiza en forma preventiva y no predictiva. En este trabajo se desarrolla un modelo matemático que describe el comportamiento de las prensas en estas máquinas papeleras. A través de simulaciones numéricas se resuelve la ecuación del movimiento obtenida del modelo propuesto y se comparan con mediciones realizadas en máquinas industriales. Se concluye que el modelo planteado describe adecuadamente el comportamiento vibratorio de las prensas y proporciona los síntomas vibratorios que permiten diagnosticar los problemas que puedan tener.
\end{abstract}

Palabras clave: análisis de vibraciones, modelo dinámico, mantenimiento predictivo, máquinas papeleras

\section{Dynamic Model of the Press Nip of Paper Machines}

\begin{abstract}
The objective of this paper is to provide the physical bases that allow more accurate diagnosis of the conditions of paper machines for better maintenance. The diagnostic of the mechanical condition of press felts and rolls in the press section of paper machines using the classical vibration analysis is complex, so maintenance of these elements is done in a preventive and not predictive way. In this work a mathematical model that describes the dynamic behavior of the presses in paper machines is developed. Through numerical simulations, the motion equation of the proposed model is solved and compared with measurements done in industrial machines. It is concluded that the proposed model properly describes the vibratory behavior of presses and provides the vibratory symptoms that allow the diagnosis of the problems that they may have.
\end{abstract}

Keywords: vibration analysis, dynamic model, predictive maintenance, paper machines 


\section{INTRODUCCION}

El mantenimiento de las máquinas ha ido tomando un rol clave en la estructura de las empresas en los últimos años, debido a que influye significativamente en los costos de producción. Un correcto plan de mantenimiento busca aumentar la confiabilidad y disponibilidad de las máquinas críticas, en los procesos productivos. En esta línea, una adecuada implementación de una estrategia de mantenimiento predictivo, basado en el análisis de vibraciones y técnicas complementarias ha sido fundamental.

Sin embargo, la industria papelera ha manifestado serias complicaciones en el análisis vibratorio de sus máquinas. Esto se debe por un lado, al poco conocimiento que los usuarios poseen de ellas, y por otro lado, al complejo comportamiento dinámico de varias de ellas comparadas con los demás equipos. Un ejemplo de este tipo de máquinas son las prensas de la máquina papelera. Lo señalado anteriormente ha conducido a que actualmente se utilice una estrategia de mantenimiento preventivo en ella, a pesar de la criticidad de esta máquina, pues cualquier falla en sus rodillos o paños puede provocar una degradación en la calidad del papel producido y por ende, un rechazo de la producción. Entonces, el desafío actual es evolucionar a una estrategia de mantenimiento predictivo, basada en el análisis de vibraciones, la cual permita en todo instante poder evaluar la condición mecánica de los rodillos y paños de las prensas en las máquinas papeleras.

En la práctica, para diagnosticar la condición mecánica de las máquinas, el análisis de las vibraciones se realiza fundamentalmente en el dominio frecuencias. Para esto es necesario determinar en forma fundamentada, tanto las componentes espectrales y formas de onda que son inherentes al funcionamiento de las prensas, como aquellas componentes espectrales y formas de onda que van a generar las diferentes fallas en los rodillos y paños.

Una de las causas más comunes en la generación de vibraciones en las prensas, es el barring del paño. Se estima que un $80 \%$ de los casos de altas vibraciones en la zona de contacto es producto de esta causa (Beck 1990). El barring en el paño consiste en la formación de marcas a lo largo de él, con variaciones en su espesor. Esta condición del paño provoca una reducción abrupta en su vida útil y puede generar marcas sobre el papel, lo que se traduce en grandes pérdidas de producción.

No existen publicaciones recientes de algún modelo matemático que explique el comportamiento vibratorio de las prensas en la zona de contacto. La más reciente es el trabajo de Lopes (1993), quién plantea un modelo por elementos finitos de una prensa compuesta por dos rodillos recubiertos con goma. En su modelo no considera al paño, sólo el contacto entre rodillos, representándolo como resortes y amortiguadores ubicados en paralelo a lo largo de ellos. Publicaciones más recientes sólo explican el origen de las vibraciones en forma cualitativa. Así, Porter (2011) explica que el aumento en el nivel de vibraciones en las prensas es producto de la pérdida de la capacidad del paño de absorber agua en ciertas zonas de él. Torniven (2010), en un informe realizado para una industria papelera, explica que la presencia de discontinuidades sobre el paño genera una vibración auto-excitada, que produce un rebote de los rodillos sobre él.

El único modelo teórico publicado que tiene relación con este tema es el modelo planteado por Chen y Boos (1975). Ambos desarrollaron un modelo teórico de un grado de libertad para explicar la formación del barring en el papel en la sección de calandrias. La ecuación del movimiento obtenida del modelo fue una ecuación de Mathieu, por lo que dedujeron que las altas vibraciones generadas por el barring en el papel, se producen cuando los parámetros operacionales están en la zona de inestabilidad del sistema.

La experiencia práctica en el tema ha sido documentada en informes realizados por empresas dedicadas al rubro del análisis de vibraciones en prensas. Ellos han reportado que las altas vibraciones medidas en las prensas ante la presencia de barring en el paño, presentan muchas componentes espectrales armónicas de la frecuencia de giro del paño. Este síntoma ha sido utilizado en la práctica para diagnosticar un paño en malas condiciones, pero sin un modelo teórico que lo respalde.

El presente trabajo tiene como objetivo desarrollar un modelo matemático sencillo que sea capaz de describir, en forma fundamentada, el comportamiento dinámico de las prensas frente a un daño del paño y/o de los rodillos. Se modela a la zona de contacto entre rodillos y paño como un sistema de masas discretas, que representan a los rodillos, conectados por un elemento elástico de rigidez variable, que representa al paño. La ecuación que define el comportamiento dinámico del sistema se resuelve numéricamente utilizando el método de Newmark.

Los síntomas vibratorios, especialmente en el dominio frecuencias, que permiten diagnosticar daño en los rodillos y/o paños, son presentados en una forma que sea útil para el personal de mantenimiento predictivo de las industrias de la Celulosa y Papel. Esto permitirá, en forma fundamentada y confiable, reemplazar la 
estrategia de mantenimiento preventivo utilizada actualmente para estos elementos, por una estrategia de mantenimiento predictivo.

\section{PRINCIPIOS BASICOS DE UNA SECCION DE PRENSAS}

La sección de prensas es una parte fundamental en las máquinas papeleras, pues afecta directamente las propiedades del papel y los costos de producción. Su función básica es disminuir el contenido de agua del papel hasta la menor cantidad posible, disminuyendo los costos energéticos utilizados posteriormente en la etapa de secado.

Esta sección está compuesta por una serie de rodillos utilizados para drenar el agua desde el papel hacia un paño (que lo transporta), aplicando presión sobre él en las distintas zonas de contacto (ver figura 1). Este proceso consiste básicamente en la compresión del papel ejercida por dos rodillos (formando la zona de contacto) para exprimir el agua del papel. Si el paño resulta saturado con agua, ésta es almacenada dentro de las ranuras o agujeros de la superficie de los rodillos bajo los paños. Dependiendo de los requerimientos del drenaje, algunas prensas utilizan cámaras de vacío para extraer el agua del paño. Así, en esta sección existen dos factores fundamentales: los paños, que son los encargados de llevar el papel a través de las distintas prensas; y la zona de contacto entre los rodillos y paño (Nip), donde el agua es transferida desde el papel al paño.

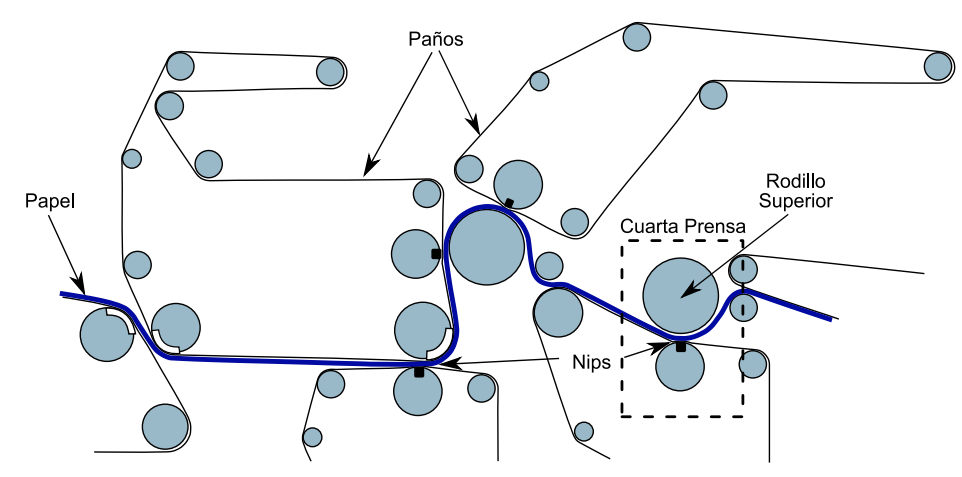

Fig. 1: Esquema de la sección de prensas de una máquina papelera.

\section{MODELO DINAMICO DE LA ZONA CONTACTO}

Un paño que va acumulando horas de funcionamiento inevitablemente disminuye su espesor, producto de la compresión ejercida por los rodillos en la zona de contacto. Esta pérdida de espesor, sumado a que el paño pierde permeabilidad con el tiempo, provoca una reducción en su volumen libre (capacidad del paño de absorber agua). En consecuencia, con el tiempo el paño presentará varias zonas en su largo con exceso de agua. Entonces, el agua al ser incompresible, formará una interfaz sólida de cuñas de agua entre los rodillos, en la zona de contacto.

Con el tiempo, las vibraciones generadas por las cuñas de agua forman marcas sobre la superficie del paño, lo que produce un aumento en la magnitud de ellas. Las marcas o barring provocan diferencias de espesor lo largo del paño. Estas variaciones de espesor producen cambios en su rigidez, afectando directamente el comportamiento dinámico de las prensas. En base a esto, se plantea un modelo simple de un grado de libertad, de la zona de contacto, apoyado en el trabajo realizado por Chen y Boos (1975), ver figura 2.

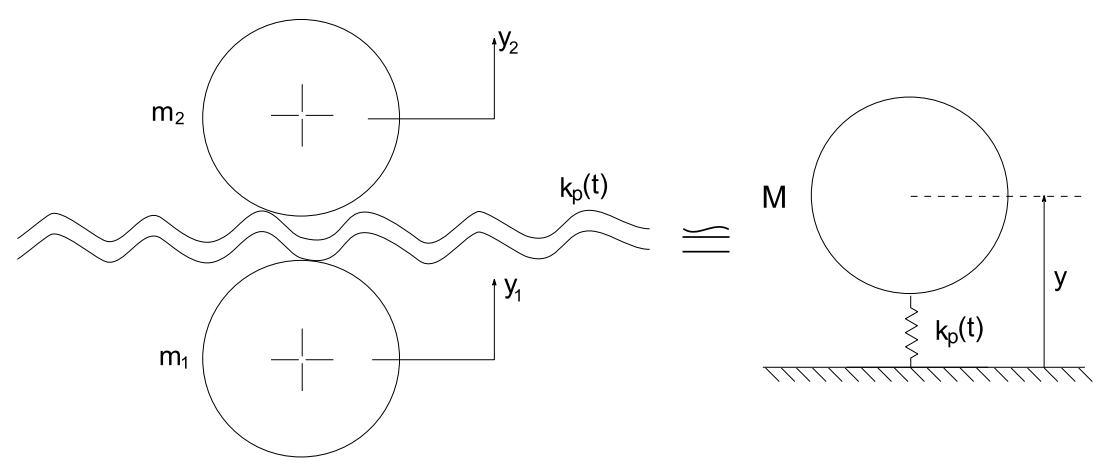

Fig. 2: Modelo para el estudio dinámico de las prensas la zona de contacto. 
Los rodillos de las prensas son modeladas como masas discretas y el paño que pasa entre ellos como un resorte de rigidez variable en el tiempo. Para simplificar el modelo se asume que el rodillo inferior tiene un desplazamiento mucho menor al superior (considerándose fijo), mientras que el superior puede moverse libremente en la dirección vertical.

En rigor, la rigidez equivalente del sistema debería considerar las rigideces del paño, papel y la de los rodillos. Sin embargo, como estos elementos están montados en serie y la rigidez del paño es bastante menor comparada a la de los rodillos y el papel, la rigidez equivalente del sistema planteado es la del elemento de menor rigidez; en este caso el paño.

\section{Modelo de la rigidez del paño}

La rigidez equivalente del sistema $k_{p}(t)$ se expresará como la suma de su valor medio " $k$ " más una parte variable generada por las diferencias en su espesor. La hipótesis formulada en el presente trabajo es definir la naturaleza de las variaciones de rigidez del paño como aleatoria, pero con una periodicidad a cada vuelta de él. Esto está basado en un principio de diseño de las prensas, que limita la longitud del paño y los perímetros de los rodillos de manera que no tengan un factor en común en sus longitudes. Si esto ocurriera, cualquier marca que se genere, por ejemplo en un rodillo, hará contacto con el otro rodillo y con el paño siempre en los mismos puntos, dañando prematuramente esos puntos de los elementos.

La selección adecuada de estas longitudes, sin factores comunes entre ellas, provoca que el contacto entre los elementos, y por lo tanto, las ubicaciones de las marcas en ellos, sean aleatorias a lo largo de cada vuelta. Por lo tanto, la variación de la rigidez del paño puede expresarse en una serie de Fourier como se indica en la ecuación 1 y se ilustra en la figura 3.

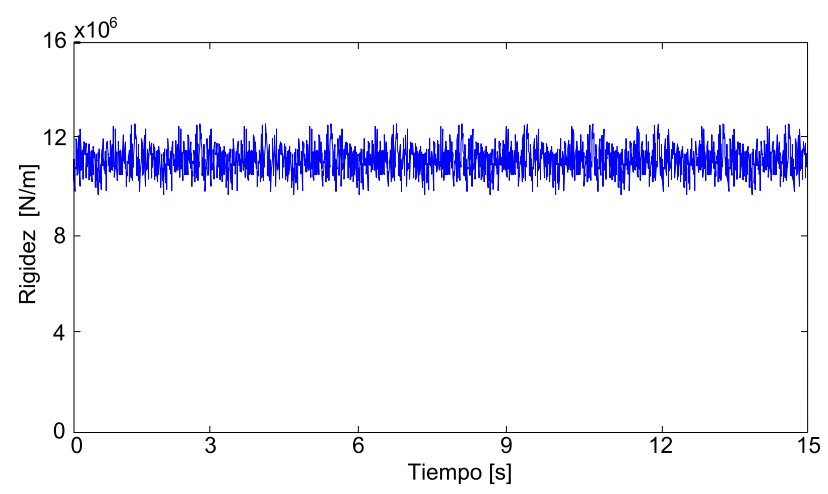

Fig. 3: Modelo de la rigidez del paño en función del tiempo.

$k_{p}(t)=k+{ }_{r=1}^{\infty} k_{r} \cos \left(2 \pi r f_{0} t+\alpha_{r}\right)$

Donde $k$ es el valor promedio de la constante de rigidez del paño $(\mathrm{N} / \mathrm{m}), k_{r}$ y $\alpha_{r}$ la amplitud y fase de la componente $r$ de la Serie de Fourier respectivamente y $f_{0}$ la frecuencia de giro del paño $(\mathrm{Hz})$.

\section{Ecuación del movimiento}

La ecuación del movimiento del modelo de la figura 2 es la siguiente:

$M y_{2}+k_{p}(t) y_{2}=M g$

Donde " $M$ " es la masa del rodillo superior $(\mathrm{kg})$, " $g$ " es la aceleración de gravedad $\left(\mathrm{m} / \mathrm{s}^{2}\right)$ e $y_{2}$ es la variable utilizada para definir el movimiento del rodillo, medida desde el punto donde el resorte tiene su largo natural.

Para simplificar la ecuación 2 , es costumbre en la literatura técnica, utilizar otra coordenada " $y$ ", relacionándola con la variable $y_{2}$ a través de una constante " $c$ ":

$y=y_{2}+c$

Cuando la rigidez del sistema es constante (de valor $k_{p}(t)=k$ ), se utiliza un valor de " $c$ " igual a la deformación estática del resorte debido al peso $(c=-M g / k)$, con lo que la ecuación 2 queda en su forma más sencilla. 
$M y+k y=0$

Sin embargo, cuando la rigidez del sistema es variable, al realizar el cambio de coordenadas señalado anteriormente se obtiene la ecuación 5 y no la ecuación 4. Este error fue cometido en el trabajo publicado por Chen y Boos (1975).

$M y+k+k_{r=1}^{\infty} k_{r} \cos \left(2 \pi r f_{0} t+\alpha_{r}\right) \quad y=c k+c k_{r=1}^{\infty} k_{r} \cos \left(2 \pi r f_{0} t+\alpha_{r}\right)$

De la ecuación 5 es interesante advertir que la simple traslación del origen del sistema de coordenadas, hacia su posición de equilibrio estático (utilizado frecuentemente en la literatura técnica), agrega a la derecha de la ecuación 4 un término que se puede interpretar como una fuerza externa sobre el sistema.

La ecuación 5 es una ecuación no homogénea de segundo orden con coeficientes variables. La solución general de este tipo de ecuación diferencial, al igual que otras ecuaciones diferenciales, no tiene solución analítica (Debnath 2005). Por lo tanto, en el presente trabajo la solución de ella se obtendrá utilizando métodos numéricos.

\section{Determinación experimental de la rigidez en compresión del paño}

El valor promedio de la rigidez del paño " $k$ " se obtuvo de forma experimental, a través de ensayos de compresión a distintas muestras de un paño, utilizado en la segunda prensa de una máquina papelera. Los ensayos se llevaron a cabo en una máquina de compresión MetroCom, a un paño Albany Apertech 300.

En las figuras 4 y 5 se muestran los resultados obtenidos de los ensayos realizados en muestras de paño nuevo (obtenido de la orilla) y en muestras compactadas, obtenidas del paño una vez terminada su vida operacional. La figura 4(a) muestra las curvas promedio fuerza-deformación medidas en los ensayos. Ellas muestran un comportamiento no lineal de la rigidez del paño a la compresión, en gran parte del rango de carga aplicada. La figura 4(b) muestra los resultados en un rango de cargas menor.

En la figura 4(b) se observa que incluso para una carga menor a $200 \mathrm{~kg}$ el comportamiento del paño no es lineal. Los puntos medidos se pueden interpolar usando una función cuadrática, indicada en esta figura. A través de esta expresión se calcula la deformación aproximada del paño con los $592 \mathrm{~kg}$ de carga, determinándose un valor aproximado de la rigidez promedio del paño " $k$ ", en la zona de contacto.

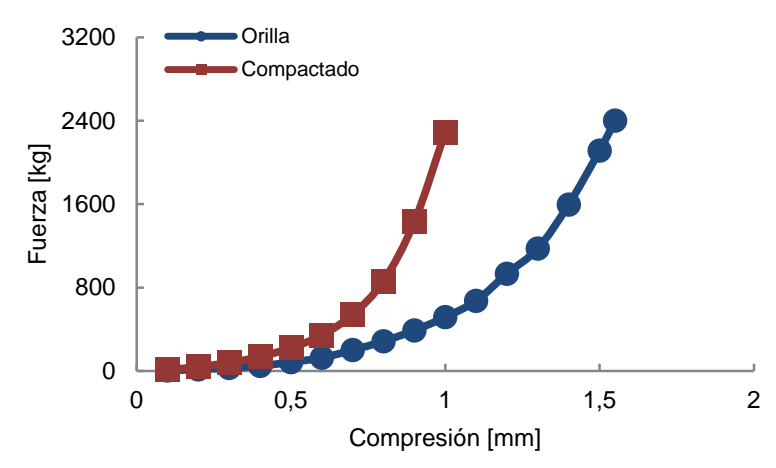

(a) Curvas fuerza-deformación del paño.

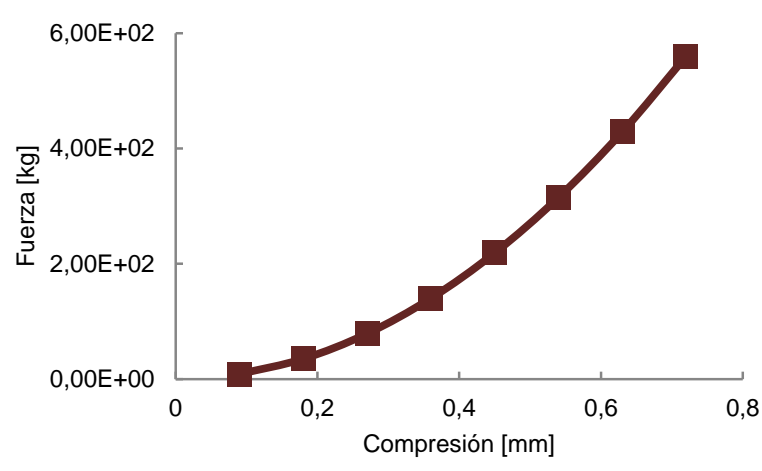

(b) Curva fuerza-deformación en el rango de interés.

Fig. 4: Curvas fuerza-deformación para el paño Apertech 300.

La rigidez determinada en estos ensayos sólo debe tomarse como un valor de referencia, pues este valor en terreno va a depender del porcentaje de agua que haya retenido el paño y fundamentalmente la fuerza de tensión con la que el paño trabaja. Estos efectos serán evaluados cualitativamente más adelante.

\section{SOLUCION NUMERICA DE LA ECUACION DEL MOVIMIENTO}

La ecuación 5 se resuelve a través de métodos numéricos. Se utiliza el método de Newmark, programado en ambiente MATLAB. Este método consiste en una integración numérica de paso simple, que determina en cada paso de tiempo el desplazamiento vibratorio a partir de la aceleración, velocidad y desplazamiento calculados en el paso de tiempo anterior. Para asegurar resultados satisfactorios con este método, se debe escoger un paso de tiempo lo suficientemente pequeño. 
Los parámetros operacionales utilizados en las simulaciones numéricas se indican en la tabla 1. La mayoría de los datos corresponden a parámetros de operación de la segunda prensa de una máquina papelera, en la región del Biobío.

Tabla 1. Datos operacionales de la Segunda Prensa MP-2.

\begin{tabular}{|c|c|c|}
\hline Parámetro & Nomenclatura & Valor \\
\hline Velocidad de Giro Paño & $f_{0}$ & $48(\mathrm{rpm})$ \\
Masa del Rodillo Cerámico & $M$ & $11000(\mathrm{~kg})$ \\
Rigidez Promedio del Paño & $k$ & $1.8 \times 10^{7}(\mathrm{~N} / \mathrm{m})$ \\
Velocidad de Rotación Rodillo & $f_{\text {rot }}$ & $318(\mathrm{rpm})$ \\
\hline
\end{tabular}

La solución numérica de la ecuación 5 , para la velocidad vibratoria del rodillo, se muestra en la figura 5. Esta simulación fue obtenida considerando la variación de rigidez del paño mostrada en la figura 2. La rigidez promedio del paño utilizada en la simulación, tiene un mayor valor que la rigidez medida en los ensayos experimentales en el laboratorio. El aumento de rigidez fue estimado considerando los porcentajes de humedad y la tensión sobre el paño con que trabaja la unidad habitualmente.

La forma de onda mostrada en la figura 5 ( a) corresponde a la respuesta total del rodillo (la parte transiente más la parte estacionaria ).La figura 5(b) muestra el espectro de la velocidad vibratoria de la figura 5(a). En él se observan varios armónicos de $48 \mathrm{rpm}$ (marcados con los cursores) y una componente (la de segundo mayor valor en el espectro) a $301 \mathrm{rpm}$. Esta última componente corresponde a la frecuencia natural del sistema y está relacionada a su respuesta transiente. Si el término a la derecha de ecuación 5 no hubiera sido considerado, esta sería la única componente vibratoria que se obtendría como solución de la ecuación, como se indica en el trabajo de Chen y Boos (1975).

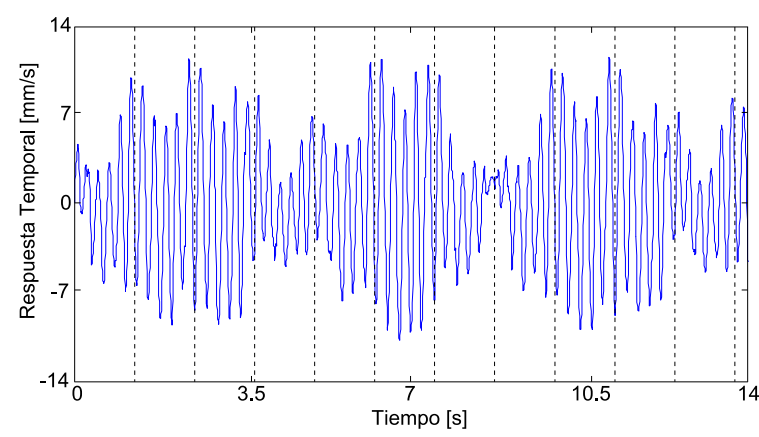

(a) Señal temporal de la respuesta total del sistema.

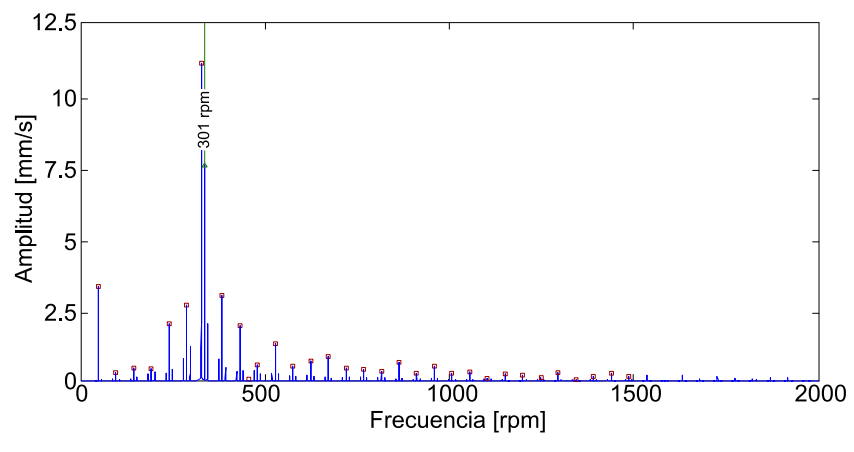

(b) Espectro de la velocidad vibratoria de la figura 5(a).

Fig. 5: Simulación numérica de la respuesta tota del sistema.

En la figura 6 se muestra la simulación numérica de la forma de onda y del espectro de la velocidad vibratoria estacionaria del cilindro. La parte transiente ha desaparecido después de un cierto tiempo por el efecto del amortiguamiento del sistema. Para ello se agregó en la ecuación del movimiento un pequeño término de amortiguamiento " $C$ " de valor arbitrario.

Analizando la respuesta estacionaria, generado por un paño dañado, se observa en la figura 6(b) que el espectro está compuesto por muchas componentes armónicas de las rpm del paño, las cuales aumentan su valor en la zona resonante del sistema (armónicos cercanos a la frecuencia natural). La forma de onda de la figura 6(a) muestra una vibración que aumenta y disminuye de valor periódicamente. Esta forma de onda puede ser generada por una modulación periódica en amplitud o por un "beat". Un "beat" es una suma de componentes cuyas frecuencias son de valores muy cercanos entre ellas, por lo que en algunos momentos estas componentes quedan casi en fase y se suman sus valores picos, mientras que en otros momentos quedan aproximadamente en contrafase, restándose sus valores. En este caso la forma de onda de la vibración ha sido generada por un "beat" (suma de componentes con frecuencias muy cercanas que se encuentran en la zona resonante).

Es interesante hacer notar que el analista de vibraciones podría pensar que el espectro de la figura 6(b) consiste en una componente de mayor valor a $4468 \mathrm{rpm}$ y varias bandas laterales separadas a la velocidad de giro del paño (48 rpm). Es importante dilucidar esto, pues ello cambia radicalmente el diagnóstico de la causa de las vibraciones. Para ello es necesario realizar un espectro con muy buena resolución y un zoom 
del espectro a bajas frecuencias (no mostrado). Con estas herramientas de análisis se demuestra, en este caso, que todas las componentes espectrales de la figura 6(b) son armónicos de la velocidad de rotación del paño, indicando un daño en él.

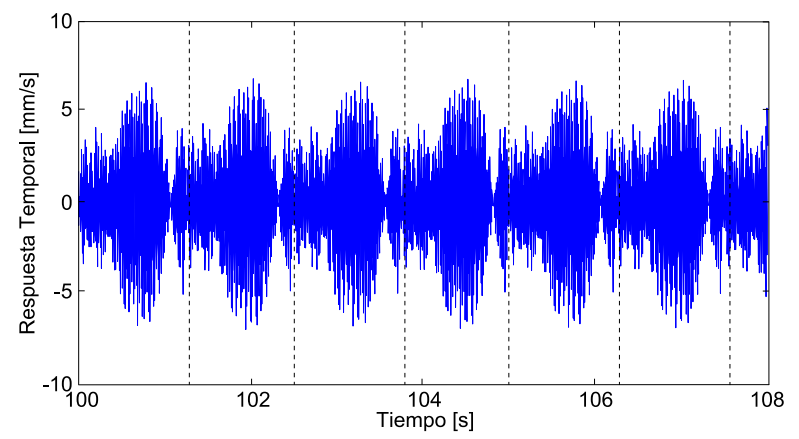

(a) Forma de onda de la vibración estacionaria.

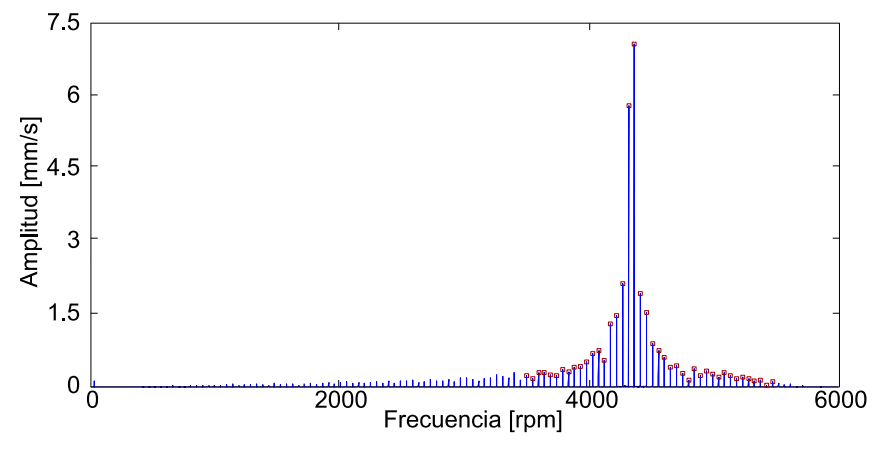

(b) Espectro vibratorio de la respuesta estacionaria.

Fig. 6: Simulación de la parte vibratoria estacionaria.

\section{Soluciones para reducir los efectos de un paño dañado}

Uno de los mayores problemas que causa el barring del paño es un alto nivel de vibraciones en las prensas, alcanzando valores inaceptables de acuerdo a las normas de severidad vibratoria y generando una calidad de fabricación del papel deficiente. Cuando sucede esto, es necesario cambiar el paño por la producción de un papel de mala calidad, con los consecuentes costos por pérdida de producción. Por lo tanto, es de interés en la industria de celulosa y papel buscar soluciones que aumenten la vida operativa de los paños.

La solución trivial para disminuir la alta magnitud de las componentes vibratorias que se encuentran en la zona resonante, generadas por un paño desgastado, es aumentar el amortiguamiento de los elementos que conforman la zona de contacto.

En la figura 7 se ilustra lo anterior. Esta figura muestra los resultados obtenidos al agregar un término "C" de amortiguamiento a la ecuación 5 . Se simuló numéricamente varias situaciones para diferentes valores del amortiguamiento "C", desde $100.000(\mathrm{~N}=\mathrm{s} / \mathrm{m})$ hasta $1.600 .000(\mathrm{~N}=\mathrm{s} / \mathrm{m})$. Como era de esperar, las componentes vibratorias, que se encuentran en la zona resonante, disminuyen significativamente de valor a medida que el valor del amortiguamiento aumenta.

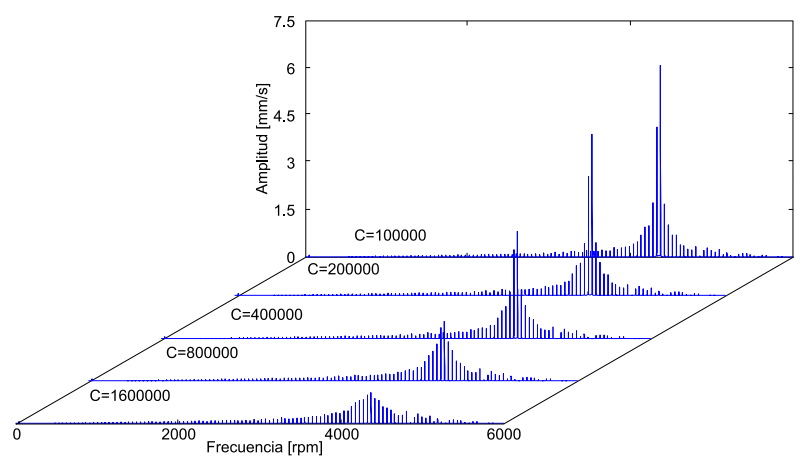

Fig. 7: Espectro en cascada para distintos valores de amortiguamiento.

Dentro de las alternativas para aumentar el valor del amortiguamiento están las siguientes opciones. La primera es agregar un sistema de amortiguamiento externo a los rodillos de las prensas, como los utilizados en industrias de diferentes rubros. Esta solución, sin embargo, es muy onerosa, por lo que existe interés en la industria del papel, en buscar otro tipo de soluciones. Sería interesante investigar si un aumento en el amortiguamiento del paño (por ejemplo, agregando una capa de poliuretano interna) genera un aumento significativo en el amortiguamiento equivalente " $C$ " de la unidad.

Una solución diferente al aumento del amortiguamiento, que se ha ensayado en algunas industrias, es tratar de disminuir la excitación que generan los cambios de espesor del paño deteriorado (término a la derecha de ecuación 5). Para ello se ha ensayado implementar sistemas automáticos que compriman las barras formadas en su largo (Porter 2011). Martínez y Portillo (2010) proponen cambiar la orientación en que las 
barras entran a la zona de contacto, obligando al paño a entrar de forma oblicua a ella, a través de un cambio de posición del rodillo tensor. No obstante, estas soluciones han permitido disminuir las vibraciones que genera el paño dañado sólo de forma temporal.

Falta por investigar soluciones más permanentes. De la ecuación 5 , se observa que el valor de la fuerza excitadora es proporcional al valor de la rigidez equivalente de la unidad. Esto deja planteado un camino de investigación sobre cómo sería factible disminuir este valor de la rigidez equivalente: por ejemplo, una solución podría ser, si los requerimientos del proceso lo permite, utilizar paños de una menor rigidez.

\section{Barring en los rodillos de las prensas}

El problema del daño en los rodillos no puede ser analizado por separado al problema de daño en los paños expuesto anteriormente. El barring en un rodillo inevitablemente dañará el paño al pasar por la zona de contacto, por lo que estos problemas serán analizados en conjunto. Una superficie con barring del rodillo es equivalente a que éste tuviera un movimiento igual a las irregularidades de su superficie $y_{r}(t)$. Con esta consideración, la ecuación del movimiento queda expresada de la siguiente forma:

$$
M\left(y-y_{r}\right)+C y-y_{r}+k+k_{r=1}^{N} k_{r} \cos 2 \pi r f_{0} t+\alpha_{r} \quad\left(y-y_{r}\right)=M g
$$

Como se busca una solución cualitativa, específicamente el contenido frecuencial de las vibraciones generadas, se utiliza para la modelación de " $y_{r}$ " una función de periodicidad igual a la velocidad de rotación del rodillo dañado, cuyo valor varía aleatoriamente en cada revolución, similar a como se modeló el barring en los paños. Para estas simulaciones se utilizaron valores promedios típicos a los valores de daño, en los rodillos medidos en terreno.

La figura 8 muestra el espectro de la velocidad vibratoria obtenido de esta última simulación. Como se esperaba están presentes los armónicos de la velocidad de rotación del rodillo dañado, marcados con los cursores, donde aquellos que están más cercanos a la frecuencia natural del sistema son de mayor valor. También están presentes armónicos de la velocidad de rotación del paño (no marcadas en el espectro) y otras componentes espectrales que no tienen relación con los armónicos de la 1xrpm paño ni con la 1xrpm del rodillo. Un análisis detallado de estas componentes, muestra que sus frecuencias son combinaciones lineales de las frecuencias anteriores. La aparición de estas componentes, torna más complejo los análisis espectrales en este tipo de máquinas, como lo señalan los analistas de vibraciones.

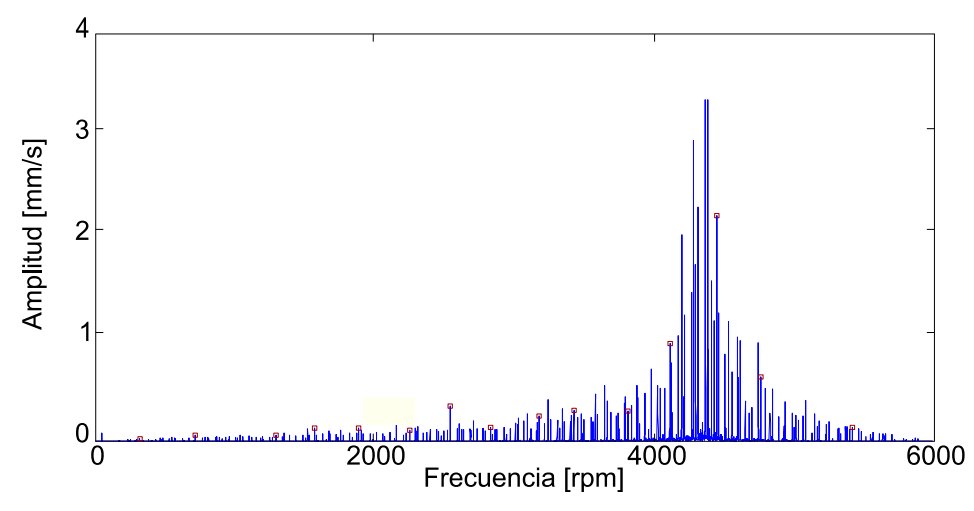

Fig. 8: Espectro vibratorio con barring en uno de los rodillos.

\section{MEDICIONES EXPERIMENTALES}

El objetivo de las simulaciones numéricas fue obtener fundamentos teóricos para interpretar con confiabilidad las mediciones reales encontradas en prensas de máquinas papeleras.

\section{Caso histórico 1}

Cuando existen varias causas que generan vibraciones, se obtienen espectros con muchas componentes espectrales, que son difíciles de analizar. Para poder separar en la vibración, que mide el sensor, sólo la vibración que genera el paño, se utiliza la técnica de los promedios sincrónicos en el tiempo. Esta técnica consiste en promediar varios registros de vibraciones, sincronizados con un pulso de frecuencia igual a la 
velocidad de giro del paño. Las componentes sincrónicas al pulso en el promedio se suman y las restantes van disminuyendo de valor con el número de promedios.

En la figura 9(b) se ve el espectro sincrónico medido en la cuarta prensa de una máquina papelera. Para obtener un pulso a cada vuelta del paño, se instaló sobre él un pedazo de tela refractante, la que se alumbró con un foto tacómetro. Con esta metodología, se realizaron 30 promedios sincrónicos de la vibración medida sobre el descanso del rodillo superior de la prensa.

Comparando las figuras 9 (a) y 9 (b) se observa que el modelo propuesto, aunque es simple, predice de forma adecuada el comportamiento vibratorio de un paño dañado. Además, se ve que los espectros simulado y medido son idénticos en su contenido frecuencial, con muchas componentes a múltiplos de la velocidad de giro del paño.

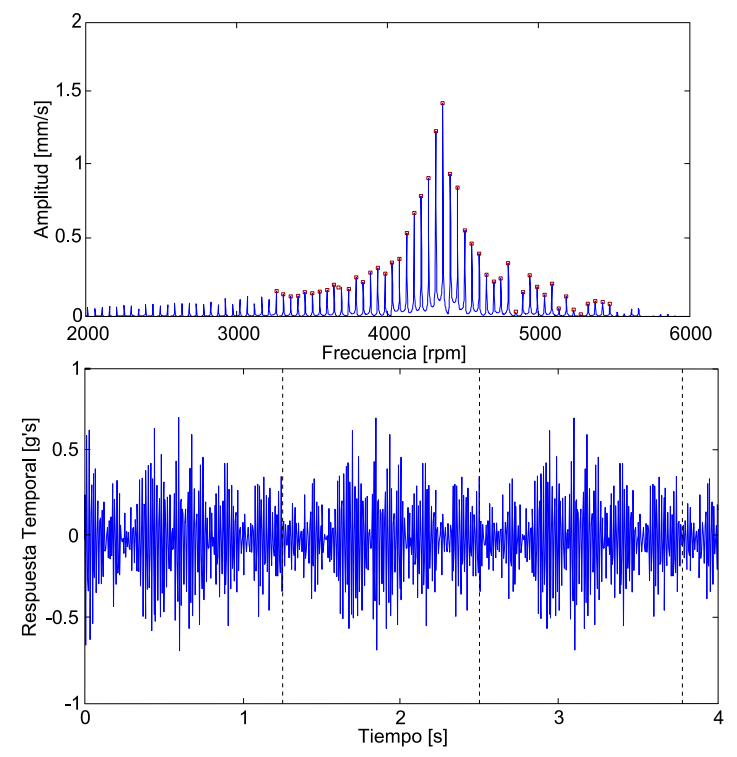

(a) Señales obtenidas de la simulación.
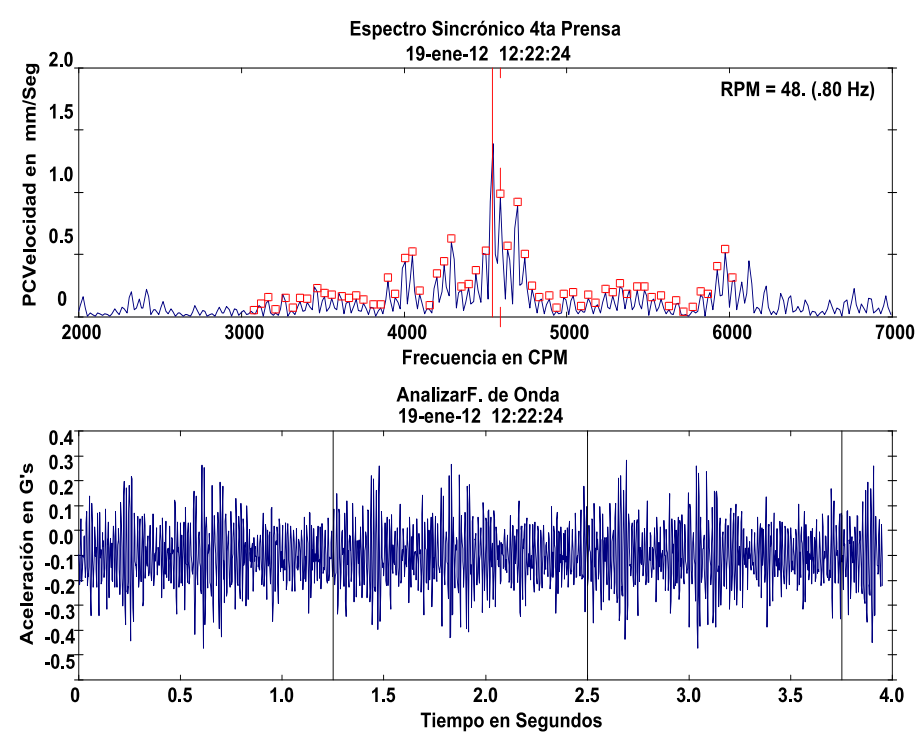

(b) Medición sincrónica en la Cuarta Prensa.

Fig. 9: Comparación entre las simulaciones y mediciones para barring en el paño.

Las formas de ondas, de la figura 9, también son similares. En ambas se aprecia los "beat" generados y la periodicidad de ellos a cada vuelta del paño (como lo indican las líneas segmentadas verticales). El "beat" en la forma de onda puede ser de gran utilidad en el diagnóstico de la condición del paño, ya que es un síntoma de la existencia de barring en él. El "beat" en la vibración genera un ruido característico parecido a un "ronroneo". Este síntoma de daño en el paño, tiene como particularidad que puede ser detectado audiblemente sin la necesidad de instrumentación.

\section{Caso histórico 2}

En este caso se analizan las vibraciones medidas en el descanso del rodillo cerámico de la segunda prensa de una máquina papelera. La figura 10(b) muestra la forma de onda y el espectro medidos. En el espectro se observan componentes espectrales múltiplos de la velocidad de rotación del rodillo cerámico (386.4 cpm), marcadas con los cursores; componentes armónicos de la velocidad de giro del paño y componentes espectrales, que como se explicó anteriormente, tienen frecuencias a combinaciones lineales de las frecuencias anteriores. En base a esto, se diagnostica un daño en el rodillo cerámico, el cual fue posteriormente cambiado.

Comparando las figuras 10 (a) y 10 (b) se observa que el espectro simulado y el medido son similares en sus contenidos frecuenciales, por lo que al igual que en el caso histórico 1, se concluye que el modelo propuesto en la ecuación 6 predice en forma adecuada el comportamiento vibratorio de un rodillo con un daño en su superficie. 

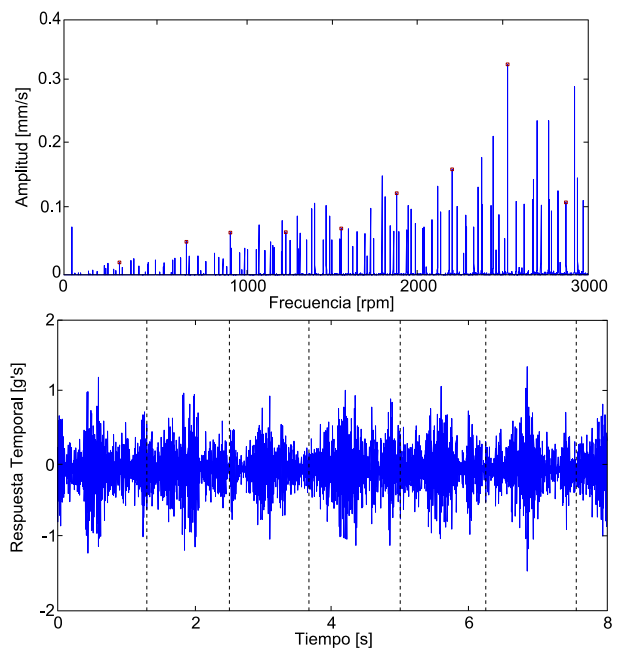

(a) Señales obtenidas de la simulación.

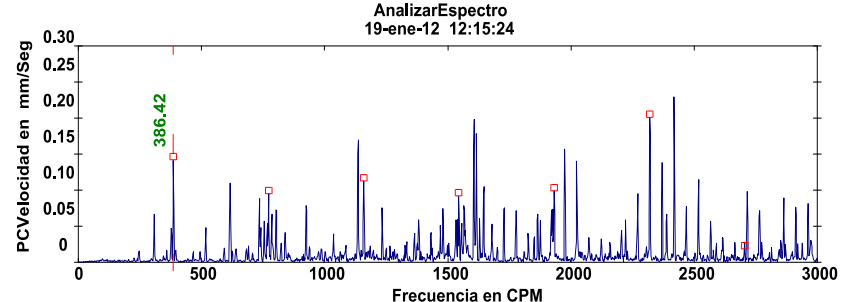

AnalizarF de Onda

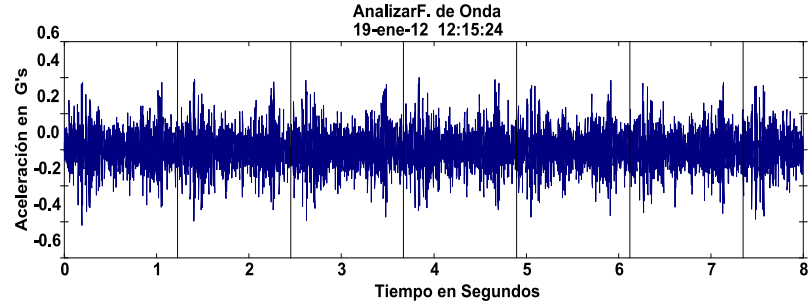

(b) Medición en la Segunda Prensa.

Fig. 10: Comparación entre las simulaciones y mediciones para barring de un rodillo.

\section{CONCLUSIONES}

El modelo teórico propuesto en el presente trabajo es adecuado para determinar la respuesta vibratoria generada por problemas frecuentes, que se producen en los paños y rodillos de las prensas de las máquinas papeleras. En los casos históricos presentados, se diagnosticaron los problemas existentes en el paño y rodillos de las prensas analizadas.

De las simulaciones efectuadas, se obtienen los síntomas vibratorios que permiten diagnosticar, mediante el análisis de vibraciones, los problemas frecuentes generados en paños y rodillos. Estos síntomas son presentados de una forma sencilla de utilizar, para el analista de vibraciones en terreno. El modelo propuesto permitió analizar el daño en rodillos y paño cuando ocurre de forma simultánea. Se obtuvo una explicación fundamentada de las componentes espectrales (que los analistas medían en terreno) y de las cuales no se conocía su origen. Del modelo, se infiere que ellas son producto de la interacción de ambos problemas y que las frecuencias de las componentes espectrales, que ello genera, son combinaciones lineales de los problemas individuales.

Conocida la física del problema, a través del modelo propuesto, se pueden buscar soluciones no tan onerosas como las que se usan actualmente, a través de mecanismos de amortiguamiento externos, para disminuir la severidad de las vibraciones que genera un paño dañado. Se propone como trabajo futuro, investigar soluciones inferidas del modelo, como aplicar amortiguamiento interno en los paños y/o disminuir la rigidez de ellos.

\section{AGRADECIMIENTOS}

Se agradece a las empresas Celulosa Arauco, Papelera Río Vergara y Papelera Norske Skog por todo el apoyo recibido para la realización de este trabajo.

\section{REFERENCIAS}

Beck, A., Finding press section vibration sources using synchronous averaging, Tappi Journal, 73(9), 199-204 (1990).

Chen, Y.N. y G. Boos, Calender barring on paper machines - Theorical model development, Tappi Journal, 58(7), 93-97 (1975).

Debnath, L., Nonlinear Partial Differential Equations for Scientists and Engineers, 3a edición, '149-185, Edinburg, Estados Unidos (2005).

Lopes V., Análise Dinâmica de Prensas de Máquinas de Papel, Tesis de Doctorado, Dpto. Proyecto Mecánicos, Universidad Estatal de Campinas, Brasil (1993).

Martínez, E. y R. Portilla, Análisis de Máquina de Papel: Sección de Prensas, Análisis de Vibraciones Heimbach-lbérica S.A., Nacimiento, Chile (2010).

Porter, S., Press Vibrations caused by Hydraulic Instability in the Nip, Paper Machine Services, Albany International Corporation, Nueva York, Estados Unidos (2011).

Torniven, A., Vibration analysis for $2^{\text {nd }}$ Press, Inc. Project No. 636/721993, Metso Paper Analysis Services, Chile (2010). 